\title{
DISTRIBUTION OF CALLICLADIUM HALDANIANUM (BRYOPHYTA, HYPNACEAE) IN POLAND
}

\author{
ADAM STEBEL
}

\begin{abstract}
Up to the mid $20^{\text {th }}$ century, Callicladium haldanianum (Grev.) H. A. Crum had been known from scattered localities, but since the 1990s the number of localities of this moss seems to have increased. At present it is a frequent or common species in eastern, central and southern Poland, whereas in the northwestern and western parts its localities have distinctly decreased in number. It is known from 209 ATMOS grid squares in total. Its highest locality, at $1407 \mathrm{~m}$ a.s.l., is in the Tatra Mts, and the lowest one is almost at sea level on the Hel Peninsula. The overwhelming majority of specimens were observed on logs and stumps. This species is frequently fertile, and about $70 \%$ of the herbarium specimens were collected with sporophytes, which often were profuse. It is not threatened in any part of Poland. A map of the current distribution of C. haldanianum in Poland is included.
\end{abstract}

Key words: Bryophyta, Callicladium haldanianum, Hypnaceae, bryogeography, distribution, Poland

Adam Stebel, Department of Pharmaceutical Botany, Medical University of Silesia in Katowice, Ostrogórska 30, 41-200 Sosnowiec; Poland; e-mail: astebel@sum.edu.pl

\section{INTRODUCTION}

Callicladium haldanianum (Grev.) H. A. Crum (Fig. 1) is a Holarctic species, in Europe having a subcontinental distribution range (Düll 1994). It is very rare from central Scandinavia to the north Mediterranean, becoming frequent towards the east (Frey et al. 2006). The situation of $C$. haldanianum in Poland is unclear. This species was reported from the present territory of Poland relatively early (e.g., Milde 1869; Limpricht 1873; Krupa 1885) but up

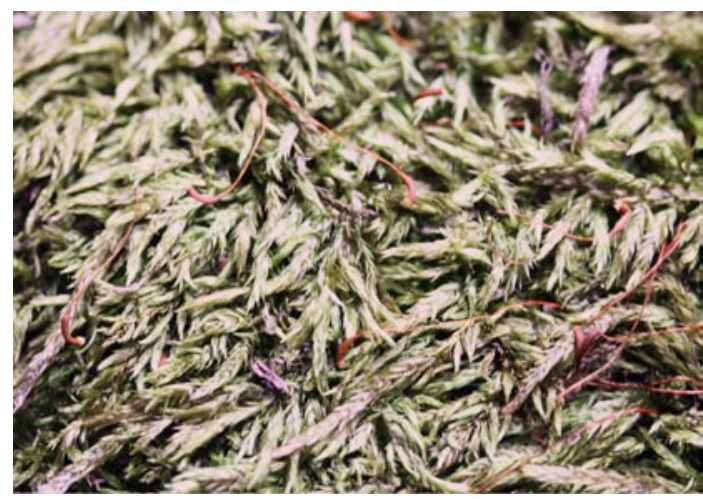

Fig. 1. Habit of Callicladium haldanianum (Grev.) H. A. Crum. to the mid $20^{\text {th }}$ century it was known from scattered localities. Szafran (1961) noted that in Poland it was relatively rare. Since the 1990s the number of localities of this moss seems to have increased. Callicladium haldanianum occurs both in natural (Fig. 2) and in managed forests (Fig. 3), where it sometimes grows in great abundance. Probably this species is spreading in Poland, like many others such as Dicranoweisia cirrata (Hedw.) Lindb., Hypnum pallescens (Hedw.) P. Beauv. and Orthodicranum tauricum (Sapjegin) Smirnova. This paper summarises all available data about the distribution and habitat preferences of Callicladium haldanianum in Poland.

\section{MATERIAL AND METHODS}

All available herbarium specimens of Callicladium haldanianum from KRAM, KTU, LBL, POZG, SOSN, WA and WRSL were revised and details of particular stations were entered in a database with the following categories: habitat, phorophyte, presence of sporophytes, and altitude. The current distribution of this species in Poland is mapped. 


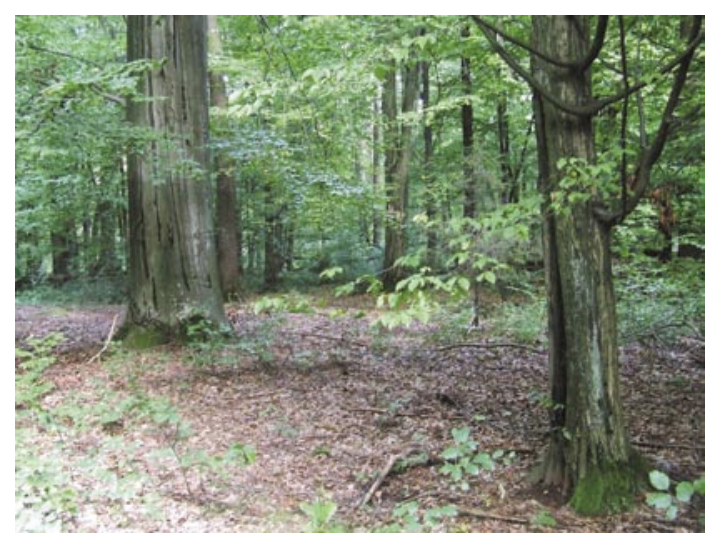

Fig. 2. Mixed forest in the Cisy koło Sierakowa Nature Reserve, Woźnicko-Wieluńska Upland (photo 7 September 2010).

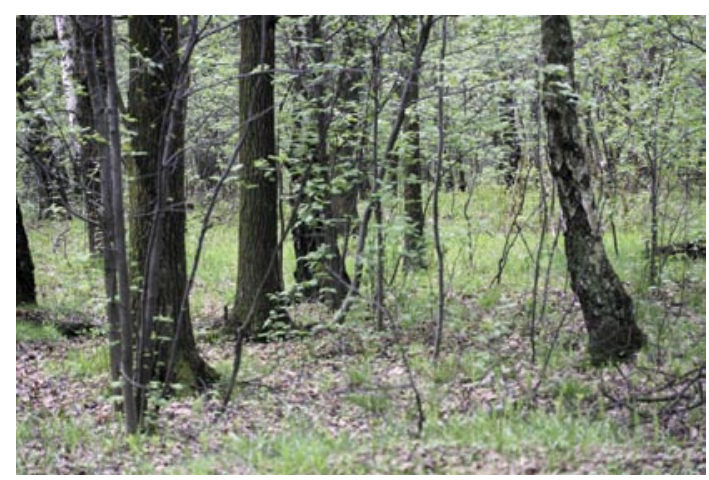

Fig. 3. Managed forest in Katowice-Osiedle Witosa, Silesian Upland (photo 5 May 2013).

Localities are given in the ATMOS cartographic system (Ochyra \& Szmajda 1981).

\section{DISTRIBUTION}

The first information about the occurrence of $\mathrm{Cal}$ licladium haldanianum in the area of present-day Poland was published by Rehmann (1865) from Kraków-Bieżanów, but the specimens housed in KRAM and WA belong to Calliergonella cuspidata (Hedw.) Loeske. A few years later, Milde (1869) published a locality from Gromnik hill (in German: Rummelsberge) in Lower Silesia collected by Hilse in 1867. During revision of herbarium materials from WRSL I found specimens recorded in 1863 in the same place by Sadebeck, and this record probably is the first one from the territory of present-day Poland. In the next years this moss was reported from dispersed localities in various regions of Poland, mainly from its eastern part (e.g., Limpricht 1873, 1904; Krupa 1882, 1885; Lisowski 1956; Kuc 1956, 1958, 1959, 1962; Karczmarz \& Kuc 1962; Lisowski \& Kornaś 1966; Szafran 1968). Since the 1990s the number of records of this species has clearly increased (e.g., Ochyra 1988; Ochyra \& Stebel 2008; Stebel 1997, 2008, 2010; Stebel \& Ochyra 2000; Fojcik \& Stebel 2001; Stebel et al. 2010; Szukalska 2007; Fojcik 2011). At present it seems to be a frequent or common species in eastern, central and southern Poland, whereas in the northwestern and western parts its localities have distinctly declined in number. Although Callicladium haldanianum is the only representative of the genus Callicladium (Crum 1971), it can be mistaken for some other moss species. In the Polish material it has been confused most often with Hypnum cupressiforme Hedw., especially with the similar in habit Hypnum cupressiforme var. subjulaceum Molendo and Calliergonella cuspidata, rarely with Platygyrium repens (Brid.) Schimp., Pylaisia polyantha (Hedw.) Schimp. and, exceptionally, with Hygrohypnum luridum (Hedw.) Jenn. The presented map of the distribution of $C$. haldanianum in Poland is based only on verified herbarium specimens (Fig. 4.). This species is known from 209 ATMOS grid squares in total.

\section{ALTITUDinAL RANGE}

Callicladium haldanianum grows both in lowland and in mountains. In mountains it occurs fairly frequently in the foothills and lower forest belt, whereas in the upper forest belt it is very rare. Its highest locality is at $1407 \mathrm{~m}$ a.s.l. near Rówień Waksmundzka in the Tatra Mts, and the lowest one is almost at sea level near Kuźnica village on the Hel Peninsula (Baltic coast).

\section{HABITATS}

The overwhelming majority of specimens (70.9\%) were collected from logs and stumps; on rocky substrate (e.g., sandstone) it was observed sporadi- 


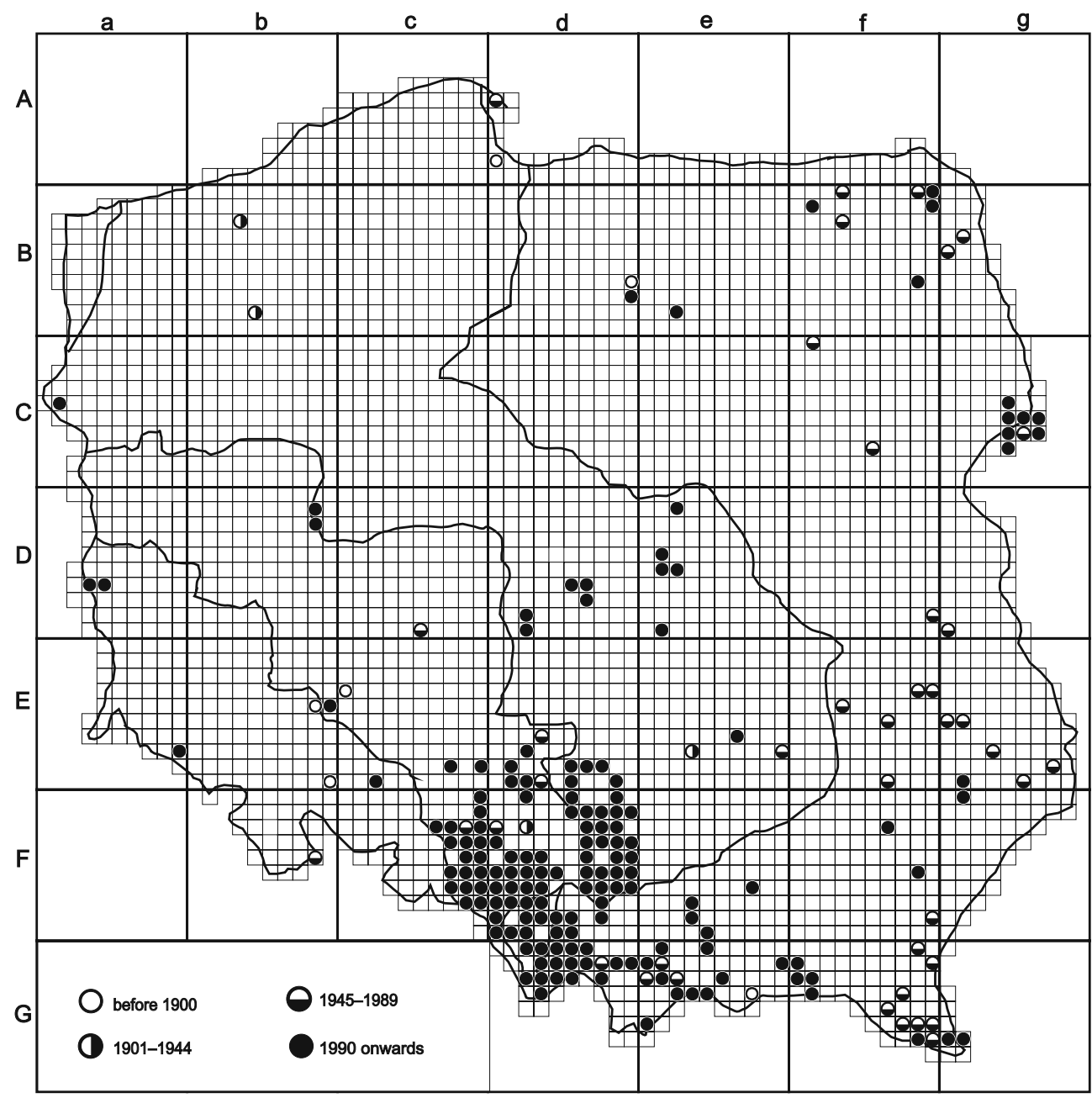

Fig. 4. Distribution of Callicladium haldanianum (Grev.) H. A. Crum in Poland.

cally (Fig. 5). Most of the oldest localities are from soil substrate, and Szafran (1961) provides such information about its ecology: 'On loamy soil in deciduous forest (mainly beech), exceptionally on logs.' Callicladium haldanianum also grows as an epiphyte, mainly at the base of trunks of deciduous trees such as Alnus glutinosa (L.) Gaertn., Quercus robur L., Betula pendula Roth and Fraxinus excelsior L. (Fig. 6).

Callicladium haldanianum forms compact green mats, frequently occupying several square meters. Particularly abundant populations occur on logs and stumps in managed forests.

\section{ACCOMPANYING SPECIES}

Callicladium haldanianum occurs in pure or mixed stands, with common bryophyte species such as Brachytheciastrum velutinum (Hedw.) Ignatov \& Huttunen, Brachythecium rutabulum (Hedw.) Schimp., Brachythecium salebrosum (F. Weber \& D. Mohr) Schimp., Herzogiella seligeri (Brid.) Z. Iwats., Hypnum cupressiforme Hedw., H. pallescens (Hedw.) P. Beauv., Lophocolea heterophylla (Schrad.) Dumort., Plagiothecium curvifolium Limpr., P. denticulatum (Hedw.) Schimp., P. laetum Schimp. and Platygyrium repens (Brid.) Schimp. 


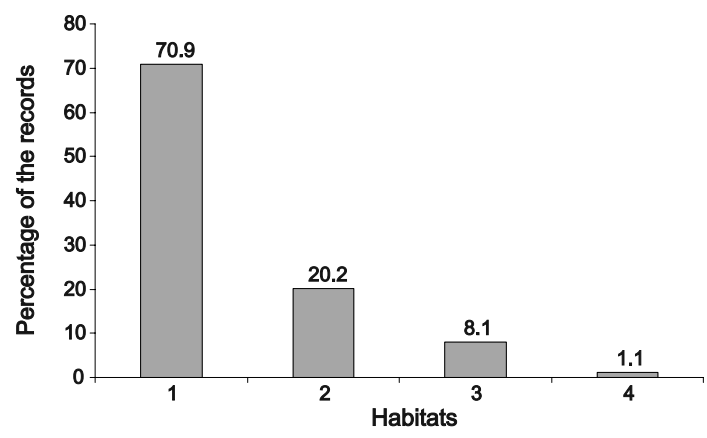

Fig. 5. Occurrence of Callicladium haldanianum (Grev.) H. A. Crum on main habitat types. 1 - epixylic, 2 - epiphytic, 3 - terrestrial, 4 - epilithic.

\section{REPRODUCTION}

In Poland Callicladium haldanianum is frequently fertile; $c a 70 \%$ of the herbarium specimens were collected with sporophytes, which often were profuse. The oldest available specimens (e.g., from Gromnik hill, Skarszyn and Wrocław-Leśnica) bear sporophytes.

\section{THREATS}

Callicladium haldanianum is not threatened in any part of Poland, but it is red-listed in the neighboring Czech Republic (vulnerable; Kučera et al. 2012) and Slovakia (vulnerable; Kubinská et al. 2001). In Germany has been classified as an extremely rare species (Meinunger \& Schröder 2007).

\section{LIST OF LOCALITIES}

Abbreviation used: f.s. - forest section No.

Grid square Ad-40 - Kuźnica, W of the village, 6 June 1981, leg. R. Ochyra (KRAM); Ad-80 - GdańskWrzeszcz, Jaśkowa Dolina, Jaśkowy Las, 18 Aug. 1891, leg. ? (POZG). Bb-23 - Białogard, 22 Oct. 1930, leg. F. Hintze (KRAM); Bb-84 - Sośnica, 27 Apr. 1904, leg. F. Hintze (LBL), 8 July 1906 \& 15 Nov. 1906, leg. F. Hintze (KRAM). Bd-69 - Ostróda, Sept. 1891, leg. Winter (KRAM); Bd-79 - Near Dylewo Nature Reserve, 14 Sept. 2004, leg. M. Szczepański (KRAM). Be-82 Źródła Łyny Nature Reserve, 19 July 2001, leg. W. Pisarek (KRAM). Bf-03 - Puszcza Borecka, f.s. 223, 14 May 1958, leg. S. Lisowski (KRAM, LBL, POZG);

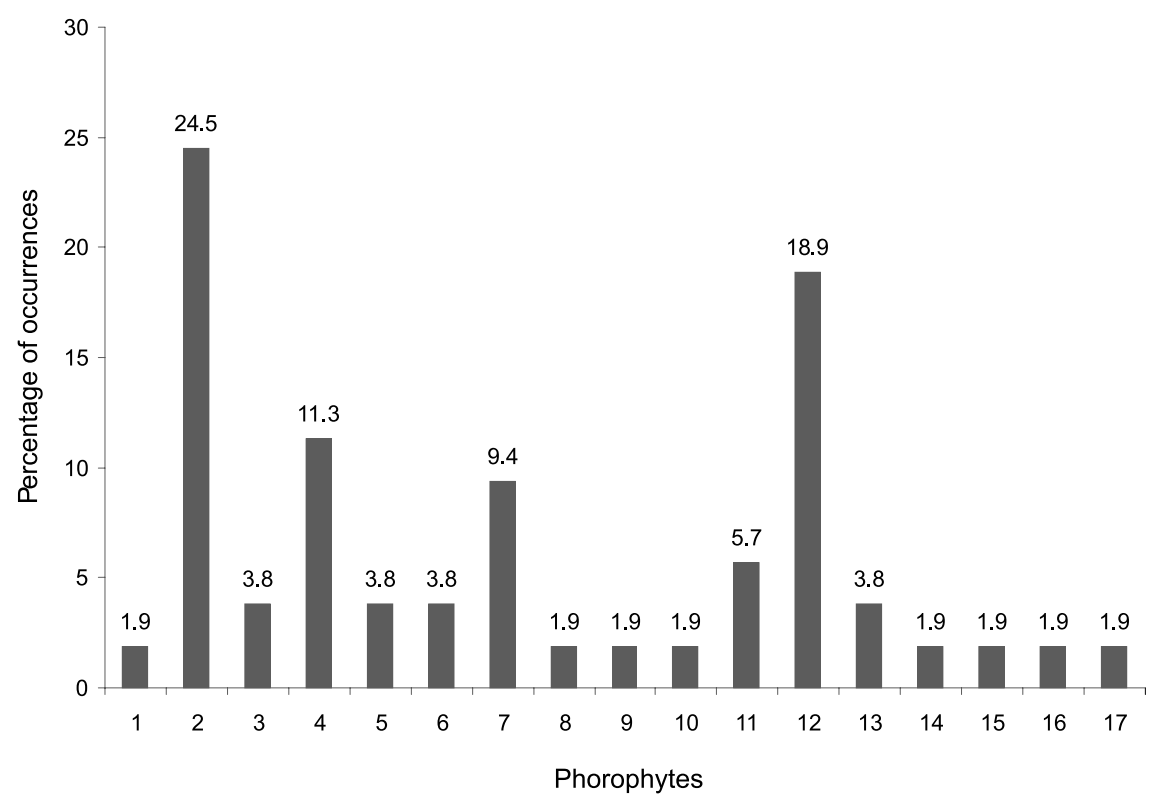

Fig. 6. Occurrence of Callicladium haldanianum (Grev.) H. A. Crum on main phorophytes in Poland. 1 - Acer platanoides, 2 - Alnus glutinosa, 3 - Alnus incana, 4 - Betula pendula, 5 - Carpinus betulus, 6 - Fagus sylvatica, 7 - Fraxinus excelsior, 8 - Platanus sp., 9 - Pinus sylvestris, 10 - Populus tremula, 11 - Populus sp., 12 - Quercus robur, 13 - Quercus rubra, 14 Quercus sp., 15 - Tilia cordata, 16 - Ulmus scabra, 17 - Ulmus sp. 
Puszcza Borecka, 4 km E of forester's lodge Czarcia Góra, 2 Aug. 1988, leg. S. Lisowski (KRAM, POZG); Bf-08 - Cmentarzysko Jaćwingów Nature Reserve, 2 Aug. 1988, leg. S. Lisowski (KRAM, POZG); Bf-09 - Wigierski National Park, Wiatrołuża river valley, f.s. 20, 9 Sept. 2012, leg. M. Staniaszek-Kik (LOD); Bf-11 - Spytkowo Nature Reserve near Giżycko, 20 July 1991, leg. B. Fojcik (KTU); Bf-19 - Wigierski National Park, Czarna Hańcza river valley, f.s. 121, 5 Sept. 2012, leg. M. Staniaszek-Kik(LOD); Bf-23 - 2 km E of Pietrosze village, 2 Aug. 1988, leg. S. Lisowski (KRAM, POZG); Bf-68 - Biebrzański National Park, Grzędy, 26 July 2011, leg. A. Stebel (SOSN). Bg-31 - Starożyn Nature Reserve, 30 Oct. 1958, leg. S. Lisowski (KRAM, POZG); Perkuć Nature Reserve, 5 Aug. 1988, leg. S. Lisowski (KRAM, POZG); Bg-40 - Kozi Rynek Nature Reserve, 4 Aug. 1988, leg. S. Lisowski (KRAM, POZG). Ca-41 - Cedyński Landscape Park, f.s. 54a, 21 June 1991, leg. E. Fudali (KRAM). Cf-01 - Łokieć Nature Reserve, f.s. 55 and 56, 21 Dec. 1982, leg. K. Karczmarz (LBL); Cf-75 - Sterdyń, Aug 1958, leg. J. Mickiewicz (WA). Cg-44 - Białowieża Primeval Forest, f.s. 60d, 20 Aug. 1992, leg. I. Melosik, A. Rusińska \& P. Urbański (POZG); Cg-54 - Dębowy Grąd Nature Reserve, f.s. 334, 12 July 1989, leg. S. Lisowski (POZG); f.s. 359d, 29 July 1991, leg. I. Melosik, A. Rusińska \& P. Urbański (POZG); Cg-55 - Białowieża Primeval Forest, f.s. 251b, 23 Aug. 1992, leg. I. Melosik, A. Rusińska \& P. Urbański (POZG), f.s. 366, 16 Aug. 1992, leg. I. Melosik, A. Rusińska \& P. Urbański (POZG), f.s. 371b, 16 March 1984, leg. A. W. Sokołowski (LBL), f.s. 395d, 16 Aug. 1992, leg. I. Melosik, A. Rusińska \& P. Urbański (POZG), f.s. 397, 12 July 1989, leg. S. Lisowski (POZG) \& f.s. 398 , 23 Apr. 1958, leg. S. Lisowski (KRAM, LBL, POZG); Białowieski National Park, f.s. 256, 15 July 1989, leg. S. Lisowski (POZG) \& 25 Apr. 1989, 26 Apr. 1989, 26 July 1989, 4 Nov. 1989, 20 June 1990 \& 21 June 1990, leg. J. Żarnowiec (SOSN), f.s. 340, 15 July 1989, leg. S. Lisowski (POZG), f.s. 372, 14 July 1989, leg. S. Lisowski (POZG) \& f.s. 399, 11 July 1989, leg. S. Lisowski (POZG); Cg-56 - Białowieża Primeval Forest, f.s. 227d, 19 Aug. 1992, leg. I. Melosik, A. Rusińska \& P. Urbański (POZG), f.s. 284, 15 July 1989, leg. S. Lisowski (POZG), f.s. 373, 14 July 1989, leg. S. Lisowski (POZG); f.s. 402, 14 July 1989, leg. S. Lisowski (POZG); Cg-64 - Białowieża Primeval Forest, f.s. 415d, 29 July 1991, leg. I. Melosik, A. Rusińska \& P. Urbański (POZG), f.s. 543b, 10 July 1981, leg. G. Wojciuk (POZG), f.s. 544a, 12 \& 17 July 1981, leg. G. Wojciuk (POZG), f.s. 465c, 13 Apr. 1983, leg. K. Karczmarz (LBL), f.s. 574c, 13 Oct. 1979, leg. K. Karczmarz (LBL), f.s. 575c, 6 Sept. 1993, leg.
A. W. Sokołowski (LBL), f.s. 576, 13 July 1989, leg. S. Lisowski (POZG), f.s. 576b, 31 Aug. 1983, leg. A. W. Sokołowski (KRAM, LBL), f.s. 602a, 22 Sept. 1983, leg. A. W. Sokotowski (KRAM, LBL), f.s. 605b, 31 Aug. 1983, leg. A. W. Sokołowski (KRAM, LBL) \& f.s. 636b, 18 Apr. 1983, leg. K. Karczmarz (LBL); Cg-65 - Białowieża, 23 June 1958, leg. J. Duda (WA); Białowieża Primeval Forest, f.s. 548c, 14 Apr. 1983, leg. K. Karczmarz (LBL); Cg-66 - Wysokie Bagno Nature Reserve, 13 Sept. 2003, leg. B. Fojcik (KTU), 13 Sept. 2003, leg. A. Stebel (SOSN) \& 13 Sept. 2003, leg. J. Żarnowiec (KRAM); Cg-74 - Białowieża Primeval Forest, f.s. 727, 17 Aug. 1992, leg. I. Melosik, A. Rusińska \& P. Urbański (POZG). Da-63 - Suchodół lake near Suchodół village, 7 Oct. 2011, leg. S. Rosadziński (POZG); Da-64 - Rzeczyca river valley near Chełm Żarski-Brody road, 21 Sept. 2012, leg. S. Rosadziński (POZG). Db-18 - Osowa Góra NW of Krosinko village, f.s. 90b, 8 Sept. 1996, leg. M. Mindur (POZG); Db-28 - Łysa Góra W of Mosina town, f.s. 142a, 7 Sept. 1996, leg. M. Mindur (POZG). Dc-95 Between Ostrów-Antonin road and Przygodziczki village, 29 May 1984, leg. W. Bocheński (POZG). Dd-65 - Zgierz, Chełmy municipal forest, f.s. 326, 20 Apr. 2001, leg. A. Łuczak (KRAM); Dd-66 - Łódź, Las Łagiewnicki, 24 June 2000, 23 Sept. 1999, 6 July 2000, 13 July 2000 \& 29 Sept. 2000, leg. A. Skrzydlak (LOD); Dd-76 - Łódź, Las Łagiewnicki, 18 Aug. 2000 \& 31 Aug. 2000, leg. A. Skrzydlak (LOD); Dd-82 - 'Jamno' Nature Reserve, 2011, leg. G. J. Wolski (LOD); Dd-92 Jabłecznik Nature Reserve, 19 Aug. 1994, leg. K. Czarna \& D. Mańkowska (LOD). De-12 - Las Łazy W of Kampinos, 24 Oct. 1969, leg. J. Mickiewicz (WA); De-41 - Bolimowski Landscape Park, Mokra, f.s. 188, 10 June 1996, leg. A. Luczak \& E. Filipiak (LOD); De-51 - Bolimowski Landscape Park, Samice, 17 July 1997, leg. A. Łuczak (LOD); De-52 - Bolimowski Landscape Park, Las Jeruzalski, f.s. 204/201, 22 July 1997, leg. A. Łuczak (LOD); De-91 - Rzeczyca commune, Struga Liciążny river valley, 23 Aug. 2010, leg. M. Majda (LOD). Df-89 - Jezioro Obradowskie Nature Reserve, 28 Aug. 1986, leg. K. Karczmarz (LBL). Dg-90 - Czarne lake near Sosnowica village, 26 May 1959, leg. S. Lisowski (KRAM, LBL, POZG). Ea-79 - Karkonosze, Długi Grzbiet, Polski Potok stream valley, $907 \mathrm{~m}$, 14 Aug. 2003, leg. M. Staniaszek-Kik (KRAM); Chojnik Mt., 558 m, 25 July 2003, leg. M. Staniaszek-Kik (KRAM). Eb-48 - Wrocław-Leśnica, 27 May 1888, leg. Schulze (WRSL); Eb-49 - Wrocław, Park Szczytnicki, 25 May 2011, leg. E. Fudali (KRAM); Eb-99 - Gromnik hill near Strzelin, NW slopes, 6 Apr. 1863, leg. Sadebeck (WRSL), Gromnik hill, Sept. 1867, leg. Hilse (KRAM, 
WA, WRSL). Ec-30 - Skarszyn, 5 Sept. 1869, leg. Schulze (WRSL) \& 21 May 1876, leg. F. Kern (WRSL); Ec-87 - Kamieniec Nature Reserve, f.s. 136g, 30 July 2008, leg. A. Stebel (SOSN); Ec-89 - Cisy koło Sierakowa Nature Reserve, 16 Aug. 2002, leg. A. Stebel, B. Fojcik \& J. B. Parusel (POZG, SOSN) \& 16 Aug. 2002, leg. B. Fojcik (KTU) \& f.s. 144g, 7 Sept. 2010, leg. A. Stebel (SOSN); Ec-92 - Kokorycz Nature Reserve, 9 Aug. 2006, leg. A. Stebel (SOSN). Ed-63Forest between Kamyk and Łobodno, 21 Dec. 1958, leg. M. Kuc (KRAM); Ed-72 - Kłobuck, Dębowa Góra, 3 Aug. 1992, leg. B. Fojcik (KRAM, KTU); Ed-81 Cisy nad Liswartą Nature Reserve, 16 Aug. 2002, leg. B. Fojcik (KTU); Cisy w Łebkach Nature Reserve, 16 Aug. 2002, leg. A. Stebel, B. Fojcik \& J. B. Parusel (POZG, SOSN) \& 16 Aug. 2002, leg. B. Fojcik (KTU); Ed-85 - Zagórze near Kobyłczyce, 11 March 2007, leg. B. Fojcik (KTU); Ed-86 - Stawki, Stawki Landscape Park, 26 June 2002, leg. B. Fojcik (KTU); Zalesice, Stawki Landscape Park, 3 July 2002, leg. B. Fojcik (KTU); Ed-87 - Borek Nature Reserve, 21 June 1989, leg. J. Żarnowiec (SOSN); Podlesie, 20 Sept. 2002, leg. B. Fojcik (KTU); Ed-91 - Forest district Kochanowice, f.s. 211j, 23 Apr. 2008, leg. P. Domagata (SOSN); Ed-92 - Rajchowa Góra Nature Reserve, 3 July 1989, leg. K. Jędrzejko (SOSN) \& 22 Aug. 2002, leg. A. Stebel \& J. B. Parusel (SOSN); Ed-93 - Between villages Młynek and Sobuczyna, 1 May 1988, leg. H. BednarekOchyra \& R. Ochyra (KRAM); Ed-95 - Parkowe Nature Reserve, near 'Diable Mosty' limestone outcrop, 17 June 2000, leg. A. Stebel (SOSN); Złoty Potok, near 'Pstragarnia', 4 Aug. 2006, leg. B. Fojcik (KTU); Ed-98 - Nakło, 1 Oct. 2002, leg. B. Fojcik (KTU). Ee-66 Świętokrzyski National Park, Czarny Las, 14 Sept. 2012, leg. B. Fojcik (KTU) \& 14 Sept 2012, leg. A. Stebel \& leg. G. Vončina (SOSN); Ee-73 - Świętokrzyskie Mts, Zgórskie range, 31 Oct. 1942, leg. K. Kaznowski (POZG); Ee-79 - Gromadzice (Zagrody?), 23 Sept. 1955, leg. M. Kuc (KRAM). Ef-38 - Kazimierzówka, 11 Sept. 1957, leg. K. Karczmarz (LBL); Ef-39 - Near Minkowice, 7 July 1957, leg. M. Kuc (KRAM); Ef-43 - Between Kaliszany, Kopanina and Świdry, 7 July 1957, leg. M. Kuc (KRAM); Ef-56 - Lipno near Wilkołaz, 7 Nov. 1957, leg. M. Kuc (KRAM); Near Polichno, 4 May 1957, leg. M. Kuc (KRAM); Ef-96 Ruda near Janów Lubelski, 15 July 1973, leg. M. Januszewska (LBL). Eg-50 - Near Orchowiec, 5 Oct. 1957, leg. M. Kuc (KRAM); Eg-51 - between Niemienice and Żółkiewka near Krasnystaw, 6 Oct. 1957, leg. M. Kuc (KRAM, LBL); Eg-73 - between Dębowiec and Żuków, 14 Sept. 1957, leg. M. Kuc (LBL); Eg-87 - Mircze, f.s. 82, 15 Sept. 1973, leg. J. Markowska
(LBL); Eg-91 - Roztoczański National Park, Bukowa Góra, 12 Sept. 1980, leg. S. Lisowski (POZG), Bukowa Góra protective district, f.s. 197, 10 Sept. 2008, leg. R. Zubel (LBL), Świerszcz stream valley, f.s. 186a, 16 Sept. 2011, leg. A. Stebel (KRAM), 16 Sept. 2011, leg. M. Staniaszek-Kik (LOD) \& f.s. 204, 16 Sept. 2011, leg. M. Staniaszek-Kik (LOD); Eg-95 - Wożuczyn, 23 March 1960, leg. K. Karczmarz (LBL). Fb-48 - Góry Bialskie, Rudawiec Mt., E slope, 850 m, 17 May 1959, leg. S. Lisowski (KRAM, POZG). Fc-09 - Zawadzkie, fish ponds in northern part, 14 June 2006, leg. A. Stebel (SOSN); Fc-19 - Hubert Nature Reserve, 10 Nov. 1996, leg. A. Stebel (KRAM, POZG, SOSN); Kielcza, Wiosenna Street, 18 June 2005, leg. A. Stebel (SOSN); Płużnica Nature Reserve, 26 Apr. 2002, leg. A. Stebel (SOSN); Fc-26 - Jasiona, 3 May 2002, leg. A. Stebel (SOSN); Łęg Zdzieszowicki, f.s. 101, 17 May 2006, leg. A. Stebel (SOSN); Fc-27 - Boże Oczko Nature Reserve, 10 June 2006, leg. A. Stebel (SOSN); Góra Św. Anny, 10 Dec. 1954, leg. M. Kuc (KRAM); Łąki Kozielskie, 2 Jan. 1998, leg. A. Stebel (SOSN); Fc-28 - Zimna Wódka, 9 May 1955, leg. M. Kuc (KRAM); Fc-29 - Widów Mały, 9 Apr. 2002, leg. A. Stebel (SOSN); Fc-37 - Stare Koźle, 30 Dec. 1997, leg. A. Stebel (SOSN); Fc-38 - Rudziniec, 27 Jun. 2000, leg. A. Stebel (SOSN); Stara Kuźnia, 16 Oct. 1996, leg. A. Stebel (KRAM, POZG, SOSN); Fc-39 - Kleszczów, 19 Aug. 1995, leg. A. Stebel (KRAM, LBL, POZG, SOSN); Kozłów-Podlesie, 6 July 2001, leg. A. Stebel (SOSN); Pławniowice, 27 June 2000, leg. A. Stebel (SOSN); Pławniowice-Rożek, 5 July 1996, leg. A. Stebel (KRAM, POZG, SOSN); Rachowice, W part, 29 March 2002, leg. A. Stebel (SOSN); Fc-48 - Kotlarnia, 27 Dec. 1997, leg. A. Stebel (SOSN); Fc 49 - Choryńskowice, 28 Dec. 1997, leg. A. Stebel (KRAM, LBL, POZG, SOSN); Rachowice, S part, 29 March 2002, leg. A. Stebel (SOSN); Stanice-Górniki, 10 Nov. 2008, leg. A. Stebel (SOSN); Tworóg Mały, NE part, 1 Jan. 1998, leg. A. Stebel (SOSN); Tworóg Mały, W part, 11 May 1994, leg. A. Stebel (KRAM, SOSN); Fc-57 - Łężczok Nature Reserve, 15 Apr. 1994, leg. A. Stebel (KRAM, SOSN); Nędza, 26 Apr. 1994, leg. A. Stebel (KRAM, SOSN); Fc-58 - Zwonowice, 29 July 1993, leg. A. Stebel (KRAM, LBL, POZG, SOSN); Fc-59 - 'Głębokie Doły' projected nature reserve, 24 Apr. 1998, 7 June 1998, 29 Apr. 2005 \& 14 May 2005, leg. A. Stebel (SOSN); Rybnik-Ochojec, f.s. 73, 28 Aug. 1994, leg. A. Stebel (KRAM, SOSN), f.s. 108, 14 March \& 31 Aug. 2005, leg. A. Stebel (SOSN) \& place called 'Anglik', 29 Apr. 2005, leg. A. Stebel (SOSN); Rybnik-Gzel, 11 Aug. 1994, leg. A. Stebel (KRAM, SOSN); Fc-67 - RacibórzObora, 14 Oct. 1993, leg. A. Stebel (KRAM, SOSN); 
Fc-68 - Lyski-Kamionki, 22 May 1993, leg. A. Stebel (KRAM, SOSN); Rydułtowy, Las Agnieszka, 19 Oct. 1992 \& 26 Sept. 2007, leg. A. Stebel (KRAM, SOSN); Fc-69 - Rybnik-Kolonia Beata, 19 Oct. 1992, leg. A. Stebel (KRAM, SOSN); Fc-78 - Wytrzęsów near Gorzyce, 23 Aug. 1993, leg. A. Stebel (KRAM, SOSN); Fc-79 - Marklowice Górne, 1 May 1993, leg. A. Stebel (KRAM, SOSN); Skrzyszów, 6 May 1994, leg. A. Stebel (KRAM, SOSN). Fd-02 - Góra Grojec Nature Reserve, 30 Apr. 2009 \& 30 Apr. 2010, leg. A. Stebel (SOSN); Fd-05 - Postęp, near the Warta river, 24 Apr. 2009, leg. A. Stebel (SOSN); Przewodziszowice near Żarki, 27 July 2003, leg. B. Fojcik (KTU); Fd-08 - Podkaszczor near Rokitno, 25 Sept. 2003, leg. B. Fojcik (KTU); Fd-15 - Zawiercie-Marciszów, 27 Apr. 2009, leg. A. Stebel (SOSN); Kopaniny, f.s. 346, 27 Apr. 2009, leg. A. Stebel (SOSN); Fd-16 - Włodowice, 9 Sept. 2006, leg. B. Fojcik (KTU); Fd-17 - Dzwonowice, 14 Apr. 2005, leg. B. Fojcik (KTU); Siedliszowice near Pradła, 25 May 2007, leg. B. Fojcik (KTU); Fd-18 - Siadcza, 18 Aug. 2004, leg. B. Fojcik (KTU); Fd-19 - ŻarnowiecDziadówki Podleśne, 15 Nov. 2002, leg. A. Stebel (KRAM, POZG, SOSN); Fd-20-Księży Las, 24 \& 25 Apr. 1955, leg. M. Kuc (KRAM); Fd-22 - Bizja, 27 Aug. 1942, leg. A. Graw (KRAM); Fd-26 - Hutki-Kanki, 26 July 2006, leg. B. Fojcik (KTU); Fd-27 - Złożeniec near Wolbrom, 12 Apr. 2007, leg. B. Fojcik (KTU); Fd-28 - Syber near Pilica, 19 Aug. 2004, leg. B. Fojcik (KTU); Udórz, 20 Oct. 2006, leg. B. Fojcik (KTU); Żarnowiec-Kresy, 15 Nov. 2002, leg. A. Stebel (KRAM, POZG, SOSN); Fd-30 - Gliwice, 'Las Dąbrowa' projected nature reserve, 9 May 1998, 17 \& 24 Jan. 1999 \& 10 Apr. 1999, leg. A. Stebel (KRAM, LBL, POZG, SOSN); Gliwice-Łabędy, 5 Oct. 1995 \& 13 Sept. 1997, leg. B. Kowalczyk (SOSN); Kolonia Pyskowice, 9 Apr. 2002, leg. A. Stebel (SOSN); Fd-36 - Klucze-Osiedle, 29 June 2005, leg. B. Fojcik (KTU); Fd-37 - Pazurek Nature Reserve, 3 June 2005, leg. B. Fojcik (KTU); Fd-38 - Trzyciąż, 16 Sept. 2005, leg. B. Fojcik (KTU); Fd-39 - Ostrysz, Ostryszni ravine, 26 Oct. 2006, leg. B. Fojcik (KTU); Fd-41 - Zabrze-Makoszowy, Stawy Makoszowskie, 9 Aug. 2007, leg. A. Stebel (SOSN); Fd-42 - Katowice-Ligota, 25 Feb. 1995, leg. A. Stebel (KRAM, LBL, POZG, SOSN); Katowice, Osiedle Witosa, 17 Nov. 2012, leg. A. Stebel (SOSN); KatowicePanewniki, 23 Sept. 1999, leg. B. Fojcik (KRAM, KTU); Fd-43 - Katowice-Giszowiec, 5 Apr. 1998 \& 1 July 1999, leg. B. Fojcik (KTU) \& 9 July 2012, leg. A. Stebel (SOSN); Katowice-Kolonia Zuzanny, 16 June 1998, leg. B. Fojcik (KTU); Katowice-Muchowiec, 4 Apr. 1998, leg. A. Stebel (SOSN); Katowice-Ochojec, 21 July 1998 \& 23 Sept. 1998, leg. B. Fojcik (KTU); Ochojec Nature
Reserve, 18 March 1995 \& 14 Sept, 1996, leg. A. Stebel (SOSN); Fd-46 - Bukowno, 'Diabla Góra' projected nature reserve, 16 June 1999, leg. A. Stebel (SOSN); Płoki, 14 June 2005, leg. B. Fojcik (KTU); Fd-48 Ojcowski National Park, Młynnik, 24 July 2005, leg. B. Fojcik (KTU), Ojców, 4 Oct. 2005, leg. B. Fojcik (KTU), Sąspowska valley, 11 May 2005, leg. B. Fojcik (KTU), Wąwóz Jamki ravine, 20 July 2005, leg. A. Stebel (SOSN), Wąwóz Sokolec ravine, 11 Aug. 2005, leg. B. Fojcik (KTU) \& 7 Sept. 2006, leg. J. Żarnowiec (KRAM), Pieskowa Skała, 18??, leg. J. Krupa (KRAM) \& 31 Aug. 2005, leg. B. Fojcik (KTU); Fd-49 - Podjedle near Olkusz, 21 Sept. 2005, leg. B. Fojcik (KTU); Stoki near Skała, 21 Oct. 2005, leg. B. Fojcik (KTU); Fd-50 - Czerwionka-Leszczyny, 19 Jan. 1993, leg. A. Stebel (KRAM, SOSN); Fd-51 - Bełk, 30 Oct. 1990, leg. A. Stebel (KRAM, SOSN); Kolonia Marzankowice near Ornontowice, 3 Nov. 1992, leg. A. Stebel (KRAM, SOSN); Łaziska Górne-Brada, 24 Apr. 1992, leg. A. Stebel (KRAM, LBL, POZG, SOSN); Orzesze, f.s. 23, 24 Apr. 1992, leg. A. Stebel (KRAM, LBL, POZG, SOSN) \& f.s. 15, 13 Oct. 1992, leg. A. Stebel (KRAM, SOSN); Orzesze-Jaśkowice, 20 Oct. 1990, leg. A. Stebel (KRAM, SOSN), upper course of the Potok Jaśkowicki, 20 March 1993, leg. A. Stebel (KRAM, SOSN) \& E of railway station, 3 Oct. 1993, leg. A. Stebel (KRAM, SOSN); Orzesze-Zawiść Szklarnia, 22 Jan. 1994, leg. A. Stebel (KRAM, LBL, POZG, SOSN); Fd-52 - Katowice-Kopaniny, 28 Dec. 1998, leg. A. Stebel (SOSN); Katowice-Boże Dary, 3 Apr. 1999, leg. A. Stebel (SOSN); Katowice-Piotrowice, between Śląska and Owsiana Streets, 27 May 2012, leg. A. Stebel (SOSN); KatowicePodlesie, 4 Nov. 1999, leg. B. Fojcik (KRAM); Katowice-Podlesie, Las Gniotek, 15 Apr. 2000, leg. A. Stebel (SOSN); Łaziska Górne, Kamienna Góra, 24 Oct. 2002, leg. B. Fojcik (KTU); Tychy-Czułów, 'Jeżowa' pond, 6 June 1996, leg. A. Stebel (SOSN); Wyry, 'Jezioro Stępniak’ pond, 26 Sept. 1995, leg. A. Stebel (SOSN); Fd-53 - Katowice-Boże Dary, 23 June 1996, 21 July 1999 \& 29 July 1999, leg. B. Fojcik (KTU); KatowiceKostuchna, Szarych Szeregów Street, 27 May 2012, leg. A. Stebel (SOSN); Katowice-Murcki, Lasy Murckowskie, many localities, 26 June 1998, 2 July 1998, 16 July 1998, 29 Sept. 1999, 2 Oct. 1999, 17 Apr. 2000, 22 May 2000, leg. B. Fojcik (KTU) \& 22 Feb. 1994, 10 March 1995, 20 March 1999, 25 March 1999, 28 March 1999, 19 Sept. 1999, 24 Apr. 2000, 24 Aug. 2008, leg. A. Stebel (SOSN); Katowice-Murcki, Siagarnia, 17 June 1998, leg. B. Fojcik (KRAM); Las Murckowski Nature Reserve, 16 Nov. 1996 \& 23 Apr. 2013, leg. A. Stebel (SOSN); Ochojec Nature Reserve, f.s. 41d, 13 May 2007, leg. A. Stebel (SOSN); Płone Bagno ecological 
area, 24 Sept. 2010, leg. A. Stebel (SOSN); TychyCzułów, 'Kielc' pond, 17 June 2004, leg. A. Stebel (SOSN); Fd-54 - Chełm Śląski-Błędów, 8 Aug. 2007, leg. A. Stebel (SOSN); Mysłowice-Kosztowy, Łąki Rzutna, 29 Apr. 2011, leg. A. Stebel (SOSN); Fd-56 Dulowa, 27 Oct. 1961, leg. B. Szafran (KRAM) \& 1 Aug. 2005, leg. B. Fojcik (KTU); Młoszowa, 3 Aug. 2005, leg. B. Fojcik (KTU); Fd-57 - Buczyna hill near Tenczynek, 6 Apr. 1953, leg. M. Kuc (KRAM); Dolina Eliaszówki valley near Czerna, $16 \mathrm{Jul}$ 2006, leg. B. Fojcik (KTU); Nawojowa Góra near Krzeszowice, 31 July 2006, leg. B. Fojcik (KTU); Fd-58 - Dolina Kluczwody valley, 25 Apr. 2007, leg. B. Fojcik (KTU); Jerzmanowice, 10 May 2007, leg. B. Fojcik (KTU); Wąwóz Bolechowicki Nature Reserve, 20 July 1987, leg. H. Klama \& J. Żarnowiec (SOSN) \& 25 May 2007, leg. B. Fojcik (KTU); Ojcowski National Park, Skały Panieńskie, 12 May 2005, leg. B. Fojcik (KTU), Wąwóz Jamki ravine, 13 May 2005, leg. B. Fojcik (KTU), Wąwóz Korytania ravine, upper part, 9 May 2005, leg. B. Fojcik (KTU), 6 Sept. 2006, leg. B. Cykowska \& leg. J. Żarnowiec (KRAM) \& 6 Sept. 2006, leg. A. Stebel (SOSN); Fd-59 - Giebułtów, 9 Oct. 2008, leg. B. Fojcik (KTU); Ojcowski National Park, Wąwóz Smardzowicki ravine, 6 Sept. 2006, leg. B. Fojcik (KTU); Fd-60 Przegędza, 19 Jan. 1993, leg. A. Stebel (KRAM, SOSN); Żory-Baranowice, old park, 14 May 1997, leg. A. Stebel (SOSN); Fd-61 - Suszec-Kolonia Podlesie, 28 March 1998, leg. B. Fojcik (KTU); Woszczyce-Szybiarz, 22 Aug. 2007, leg. A. Stebel (SOSN); Fd-62 - Babczyna Dolina Nature Reserve, f.s. 122, 16 Oct. 1991, leg. A. Stebel (KRAM, SOSN), f.s. 147, 21 Apr. 1990, leg. A. Stebel (KRAM, SOSN) \& f.s. 148, 12 July 1991, leg. A. Stebel \& J. Żarnowiec (SOSN); Gostyń, 28 June 1990, leg. A. Stebel (KRAM, SOSN); Pszczyna-Piasek, 7 Oct. 1994, leg. A. Stebel (KRAM, SOSN); Suszec, f.s. 146, 22 Sept. 2010, leg. A. Stebel (SOSN); Tychy, 'Mały Lasek' ecological area, 30 July 2010, leg. A. Stebel (SOSN); Tychy, 'Paprocany' ecological area, 13 Oct. 1992, leg. J. Żarnowiec \& S. Wika (SOSN); Wyry, 'Stary Staw' projected ecological area, 26 Sept. 1995, leg. A. M. Stebel \& A. Stebel (SOSN); Fd-63 - Międzyrzecze, f.s. 29, 12 July 1993 \& 30 June 1994, leg. A. Stebel (KRAM, SOSN); Żubrowisko Nature Reserve, f.s. 54d, 23 Sept. 1997, leg. A. Stebel (SOSN); Fd-66 - Kwaczała, Gródek ravine, 29 May 2008, leg. B. Fojcik (KTU); Nieporaz, Puszcza Dulowska, 28 Sept. 2008, leg. B. Fojcik (KTU); Fd-67 - Poręba-Żegoty, 20 July 2006, leg. B. Fojcik(KTU); Tenczynek, 2 Aug. 2005, leg. B. Fojcik (KTU); Fd-68 - Kraków-Balice, 7 May 2006, leg. B. Fojcik (KTU); Kraków-Bielany, 13 Oct. 2008, leg. B. Fojcik(KTU); Zabierzów, 11 Jul 2006, leg. B. Fojcik
(KTU); Fd-69 - Kraków, Las Wolski, 4 May 2007, leg. B. Fojcik (KTU) \& 13 Jan. 2008, leg. J. Larrain \& B. Cykowska (KRAM); Las Wolski, Sowiniec hill, 20 Aug. 1989, leg. R. Ochyra (KRAM, POZG, SOSN); Panieńskie Skały Nature Reserve, Nov 1879 \& Nov. 1880, leg. J. Krupa (KRAM) \& 23 July 1987, leg. H. Klama, K. Jędrzejko \& J. Żarnowiec (SOSN); Rząska, 5 May 2006, leg. B. Fojcik (KTU); Wola Justowska, Las Wolski, 4 May 2007, leg. B. Fojcik (KTU); Fd-70 - Bzie Górne, 5 July 1993, leg. A. Stebel (KRAM, SOSN); Fd-71 - Pszczyna-Studzionka, Czarne Doły, 9 May 2005, leg. A. Stebel (SOSN); Fd-72 Pszczyna, Park Zamkowy, 26 Apr. 1992, 12 \& 13 Oct. 1992, 17 Jan. 1993 \& 13 Sept. 1995, leg. A. Stebel (KRAM, LBL, POZG, SOSN); Fd-73 - Góra, Zapadź ecological area, 30 June 2012, leg. A. Stebel (KRAM, SOSN); 77 - Przeginia Duchowna, 20 Apr. 2006, leg. B. Fojcik (KTU); Fd-80 - Jarząbkowice, 24 June 1993 \& 26 June 1993, leg. A. Stebel (KRAM, LBL, POZG, SOSN); Pruchna, Las Makowina, 24 June 1993, leg. A. Stebel (KRAM, LBL, POZG, SOSN); ZebrzydowiceOwczarnia, 27 July 1993, leg. A. Stebel (KRAM, SOSN); Fd-82 - Czechowice-Dziedzice Krzywa, 290 m, 9 Sept. 1993, leg. K. Jędrzejko \& A. Stebel (KRAM, LBL, POZG, SOSN); Goczałkowice-Zdrój, 24 June 1993, leg. A. Stebel (KRAM, SOSN); Fd-83 - Bestwina, 29 June 1998, leg. B. Fojcik (KRAM, KTU); Janowice, Las Janowicki, 295 m, 4 May 1994, leg. K. Jędrzejko \& A. Stebel (KRAM, LBL, POZG, SOSN); Fd-84 Harszówki Dolne, 295 m, 10 Sept. 1993, leg. K. Jędrzejko \& A. Stebel (KRAM, LBL, POZG, SOSN); Harszówki, 300 m, 8 Oct. 1991, leg. K. Jędrzejko, H. Klama, J. Żarnowiec \& A. Stebel (KRAM, LBL, POZG, SOSN); Fd-85 - Andrychów, Pańska Góra Mt., NW slope, 375-390 m, 29 March 2010, leg. A. Stebel (KRAM, SOSN); Fd-87 - Bugaj, S part, 342 m, 1 Jan. 2010, leg. A. Stebel (SOSN); Fd-90 - Las Parchowiec, W part, 290-300 m, 24 Feb. 2008, leg. A. Stebel (SOSN) \& E part, 295-300 m, 24 Feb. 2008, leg. A. Stebel (SOSN); Fd-91 - Skarpa Wiślicka Nature Reserve, 315 m, 14 Sept. 1999, leg. A. Stebel (SOSN); Fd-92 Morzyk Nature Reserve, 335-345 m, 14 Sept. 1999, leg. A. Stebel (SOSN); Wapienica, NW slope of Szyndzielnia Mt., between Barbara and Żydowski streams, 600-620 m, 26 July 2000, leg. A. Stebel (SOSN); Fd-94 - Kobiernice-Łazy, 357 m, 29 March 2010, leg. A. Stebel (SOSN); Przełęcz Przysłop, 808 m, 9 Apr. 2010, leg. A. Stebel (SOSN); Szeroka Nature Reserve, Aug. 1995, leg. J. Żarnowiec \& H. Klama (KRAM); Wielka Puszcza stream valley, $390 \mathrm{~m}$, 20 March 2010, leg. A. Stebel (SOSN); Fd-95 - KocierzWalaszki, 550 m, 28 July 1996, leg. A. M. Stebel 
\& A. Stebel (KRAM, SOSN). Fe-67 - Tarnów, Park Strzelecki, 15 July 1995, leg. R. Kempa (KRAM); Fe-73 - Kopaliny, Las Kopaliński, 330-340 m, 18 Aug. 2012, leg. A. Stebel (SOSN); Fe-83 - Kamienie Brodzińskiego nature monument, W slope, 395-410 m, 25 Aug. 2012, leg. A. Stebel (SOSN); Lipnica Górna-Granica, springs of the Górzański Potok stream, 370-400 m, 25 Aug. 2012, leg. A. Stebel (SOSN); Fe-94 - Będzieszyna, 335 m, 9 Jun 1998, leg. A. Stebel (SOSN); Witowice Górne, W slope of Ostra Góra Mt., 425 m, 28 Aug. 1997, leg. A. Stebel (KRAM, POZG, SOSN). Ff-26Łętownia, near railway station, 11 May 1998, leg. A. Gumieniak (KTU); Ff-58 - Jagiełla near Przeworsk, 25 Dec. 2000, leg. R. Ochyra (KRAM); Ff-89 - Hołubie (= Hołubla near Wapowce), 16 June 1965, leg. B. Szafran (KRAM); Wesołówka, 16 June 1965, leg. B. Szafran (KRAM). Fg-01 - Roztoczański National Park, Świerszcz stream valley, f.s. 290b, 15 Sept. 2011 leg. A. Stebel (KRAM) \& 15 Sept. 2011, leg. M. StaniaszekKik (LOD). Gd-02 - 'Stok Szyndzielni' Nature Reserve, 800-820 m, 26 July 2000, leg. A. Stebel (SOSN); Gd-03 - Bułgaria, Biała stream valley, 415-425 m, 30 Apr. 2000, leg. A. Stebel (SOSN); Kalna, Kalonka stream valley, 395-400 m, 16 Apr. 2003, leg. A. Stebel (SOSN); Gd-04 - Czernichów-Surkowie, 520 m, 5 Aug. 1995, leg. A.M. Stebel \& A. Stebel (SOSN); 'Grapa' Nature Reserve, 420-425 m, 1 Sept. 1999, leg. A. Stebel (SOSN); Żywiec-Moszczanica, between the Moszczanica stream valley and Żywiec-Kocurów, 380-390 m, 22 Apr. 2003, leg. A. Stebel (KRAM, POZG, SOSN); Żywiec-Moszczanica, old park, 365-370 m, 16 June 2000, leg. A. Stebel (SOSN); Gd-05 - Hucisko, upper course of the Pewelica stream, 545-550 m, 20 Sept. 2003, leg. A. Stebel (KRAM, POZG, SOSN); Kucówki, 840 m, 2 Aug. 1996, leg. A. Stebel \& A.M. Stebel (SOSN); Przełęcz pod Płonem, 690 m, 12 Aug. 1995, leg. A. Stebel (KRAM, POZG, SOSN); ŚlemieńMłyńsko, Młyńska Rzeka stream valley, 510-515 m, 27 Aug. 2002, leg. A. Stebel (SOSN); Gd-06 - Lachowice-Buczanka, 580-590 m, 7 Aug. 2003, leg. A. Stebel (SOSN); Gd-13 - Barania Góra Nature Reserve, Biała Wisełka stream valley, 1050-1055 m, 4 Sept. 2002, leg. A. Stebel (SOSN); Gd-14 - Duży Grojec Mt., NW slope, 430 m, 13 May 2006, leg. A. Stebel (SOSN); Gd-15 between Sopotnia Wielka and Sopotnia Mała, 530535 m, 17 Aug. 2004, leg. A. Stebel (SOSN); Gd-16 - Babia Góra massif, Zawoja-Markowa, towards Pośredni Bór, 730 m, 2 Sept. 1997, leg. A. Stebel (SOSN); Polica massif, Zawoja-(Mosorne)Podgórze, 610 m, 27 July 1978, leg. R. Ochyra (KRAM); Mędralowa, W part, 1080 m, 19 Aug. 2003, leg. A. Stebel (SOSN); Przełęcz Opaczne, 935 m, 26 Aug. 2003, leg.
A. Stebel (KRAM, SOSN, POZG); Gd-17 - Polica massif, between Policzne and Przełęcz Krowiarki, 740 m, 1966, leg. L. Stuchlik \& M. Kuc (KRAM); Gd-18 - Wysoka, Pożoga stream valley, 517-522 m, 1 May 2012, leg. A. Stebel (SOSN); Gd-19 - between Jawornik Mt. and Góra Ludwiki Mt., 615-622 m, 1 May 2012, leg. A. Stebel (SOSN); Gd-22 - Jaworzynka, Olecka stream valley, 555-560 m, 24 May 2001, leg. A. Stebel (SOSN); Gd-23 - Hutyrów, N slope, 580-600 m, 26 Nov. 1995, leg. B. Kowalczyk (SOSN); Gd-24 Żabnica-Skałka, Studziański stream valley, $640-645 \mathrm{~m}$, 15 July 2003, leg. A. Stebel (SOSN); Gd-25 - Pilsko massif, below Hala Buczynka, 950 m, 5 May 1957, leg. S.Lisowski (KRAM, LBL, POZG); Kobielów, Kamienna stream valley, 785-790 m, 12 Aug. 2000, leg. A. Stebel (SOSN); Gd-27 - Habzów Mt., S slope, 790-800 m, 1 May 2011, leg. A. Stebel (SOSN); Gd-33 - Szczytkówka, NW part, 825-850 m, 25 July 1996, leg. B. Kowalczyk (SOSN) \& E part, 750-770 m, 18 July 1996, leg. B. Kowalczyk (SOSN). Ge-01 - Śnieżnica Mt., N slope, 710-725 m, 14 Aug. 1999, leg. A. Stebel (SOSN); Ge-04 - Białowodzka Góra Nature Reserve, NW part, 535-540 m, 2 May 2003, leg. A. Stebel (SOSN); Ge-10 - Luboń Wielki Mt., S slope, 610 615 m, 26 June 2000, leg. A. Stebel (SOSN); Ge-11 Kudłoń Mt., S slope, 1030 m, 3 Apr. 1959, leg. S. Lisowski (POZG); Ge-19 - Barwinek Mt., W slope, springs of the Bednarka stream, 540 m, 27 June 1998, leg. A. Stebel (KRAM); Ge-20 - Olszowy Potok stream valley, 850-900 m, 11 Aug. 1957 \& 27 Apr. 1959, leg. S. Lisowski (KRAM, POZG); Ge-21 - Forędówka, 1020 m, 1 Apr. 1959, leg. S. Lisowski (POZG); Łopuszna stream valley, 1 Apr. 1959, leg. S. Lisowski (POZG); Mostownica Mt., E slope, 1100 m, 10 Aug. 1957, leg. S. Lisowski (KRAM); Mostownica Mt., 2 Apr. 1959, leg. S. Lisowski (POZG); between Stawieniec mountain pasture and Kamienica river valley, 950 m, 11 Aug. 2003, leg. A. Stebel (SOSN); Turbacz stream valley, 800 m, 11 Aug. 1957, leg. S. Lisowski (KRAM, LBL, POZG); Ge-22 - Gorc Mt., 7 Sept. 1959, leg. S. Lisowski (POZG); Ge-25 - Wierch nad Kamieniem Mt., 1070 m, 21 Sept. 2012, leg. A. Stebel (SOSN); Ge-32 - Dolina Harczygrund valley, 600-610 m, 6 July 2008, leg. A. Stebel (SOSN); Ge-33 - Pieniński Potok stream valley, 650-660 m, 16 March \& 14 Sept. 2008, leg. G. Vončina (SOSN); Ge-34 - Baniska Nature Reserve, 11 Nov. 1969, leg. H. Mamczarz (KRAM); Wąwóz Homole Nature Reserve, near Kamionka stream, 595 m, 27 June 2007, leg. A. Stebel (KRAM, SOSN); Ge-37 - between Mochnaczka and Krzyżówka, forest in Huta near Krynica, Aug. 1877, leg. J. Krupa (KRAM); Krynica, Aug. 18??, leg. J. Krupa (KRAM); Ge-50 - 
Rówień Waksmundzka, near tourist track to Gęsia Szyja Mt., 1407 m, 1 Sept. 2012, leg. A. Stebel (SOSN); Wielka Pańszczycka Młaka, 1250-1255 m, 1 Sept. 2012, leg. A. Stebel (SOSN). Gf-08 - Posada Rybotycka, 3 \& 4 Aug. 1989, leg. H. Bednarek-Ochyra (KRAM); Gf-10 - Folusz, towards Huta Pielgrzymska, 405 m, 4 June 1999, leg. A. Stebel (SOSN); Magura Mt., 840 m, 25 June 1998, leg. A. Stebel (KRAM); Gf-19 - Jamna Mt., 15 Oct. 1983, leg. R. Ochyra \& H. BednarekOchyra (KRAM); Gf-20 - Krempna, central part of the Krempna stream valley, 445 m, 30 May 1999, leg. A. Stebel (KRAM, SOSN); Rostajne, near forester's lodge, 420-430 m, 1 May 1998, leg. A. Stebel (KRAM); Świątkowa Wielka, upper course of the Krokowy stream, 510 m, 27 Sept. 1998, leg. A. Stebel (KRAM, SOSN); Gf-21 - Las Huciska near Huta Krempska, 500 m, 30 May 1999, leg. A. Stebel (KRAM); Gf-31 - Polany, Baranie stream valley, 580-600 m, 2 May 1998, leg. A. Stebel (KRAM, POZG, SOSN); Gf-37 - Near Solina, 500 m, 18 July 1982, leg. S. Lisowski (POZG); Gf-46 - Duszatyn, Jeziorka Duszatyńskie, 700 m, 20 July 1982, leg. S. Lisowski (KRAM, POZG); Gf-57 Łupienik Mt., S slope, $850 \mathrm{~m}, 7$ Sept. 1955, leg. S. Lisowski (POZG); Gf-58 - Smerek Mt., S slope, 780 m, 1 Sept. 1955, leg. S. Lisowski (POZG); Gf-59 - Stuposiany, 9 Aug. 1955, leg. S. Lisowski (POZG); Gf-68 - Dział Mt., W slope, 750 m, 24 Apr. 1956, leg. S. Lisowski (KRAM, LBL, POZG); Moczarne, Górna Solinka stream valley, 715 m, 26 Aug. 2009, leg. A. Stebel (KRAM, SOSN); Gf-69 - Between Bereżki and Ustrzyki Górne, 650 m, 22 Sept. 1954, leg. S. Lisowski (POZG); Rzeczyca stream valley, 23 Apr. 1956, leg. S. Lisowski (POZG); Widełki, 11 July 1962, leg. M. Kuc (KRAM). Gg-60 - Terebowiec stream valley, 800-825 m, 23, 24 \& 26 July 1991, leg. J. Żarnowiec \& M. Szymocha (KRAM, SOSN) \& 875940 m, 13 June 1993, leg. J. Żarnowiec \& M. Szymocha (KRAM, SOSN); Gg-61 - Litmirz Nature Reserve, 691 m, 21 July 2009, leg. A. Stebel, J. Żarnowiec \& G. Wolański (SOSN).

ACKNOWLEDGEMENTS. I am grateful to the curators at KRAM, KTU, LBL, POZG, WA and WRSL, and to Halina Bednarek-Ochyra, Barbara Fojcik, Ewa Fudali, Ryszard Ochyra, Włodzimierz Pisarek, Stanisław Rosadziński, Anna Rusińska, Monika Staniaszek-Kik, Mirosław Szczepański, Grzegorz J. Wolski and Jan Żarnowiec for kindly allowing me to study herbarium specimens of Callicladium haldanianum. This work was supported in part by the Polish Ministry of Education and Science (MEiN) for 2010-2014 (grant No. N N303 572038).

\section{REFERENCES}

CRUM H. A. 1971. Nomenclatural changes in the Musci. Bryologist 74: 165-174.

DÜLL R. 1994. Deutschlands Moose. 3 Teil. Orthotrichales: Hedwigiaceae - Hypnobryales: Hypnaceae. IDH Verlag, Bad Münstereifel-Ohlerath.

FOJCIK B. 2011. Distribution atlas of mosses of the CracowCzęstochowa Upland. Centrum Dziedzictwa Przyrody Górnego Śląska, Katowice.

FoJCIK B. \& STEBEL A. 2001. Ecological and spatial structure of bryoflora of Katowice town. Centrum Dziedzictwa Przyrody Górnego Ślaska w Katowicach. Materialy Opracowania 5: 1-128 (in Polish with English summary).

Frey W., Frahm J.-P., Fischer E. \& Lobin W. 2006. The liverworts, mosses and ferns of Europe. Harley Books.

KARCZMARZ K. \& KUC M. 1962. The mosses of the eastern part of the Lublin Upland. Fragm. Florist. Geobot. 8(4): 483-508 (in Polish with English summary).

KRUPA J. 1882. Zapiski bryjologiczne. Sprawozdania Komisji Fizyjograficznej 16: 170-204.

KRUPA J. 1885. Zapiski bryjologiczne z okolic Lwowa, Krakowa i wschodnich Karpat. Sprawozdania Komisji Fizyjograficznej 19: 133-164.

KuBINSKÁ A., JANOVICOVÁ K. \& ŠOLTÉS R. 2001. Updated checklist of liverworts, hornworts and mosses of Slovakia. Bryonora 28: 4-10 (in Slovak with English summary).

KUC M. 1956. The mosses of the Silesian Upland (The Muschelkalk Area). Acta Soc. Bot. Poloniae 25(4): 629673 (in Polish with English summary).

KUC M. 1958. Bryological records from the Polish Tatra Mountains. Rev. Bryol. Lichénol. 25: 31-37.

KuC M. 1959. The mosses of the Sandomierz - Opatów Upland. Fragm. Florist. Geobot. 5(1): 129-150 (in Polish with English summary).

KUC M. 1962. Mosses of the western part of the Lublin Upland Fragm. Florist. Geobot. 8(1): 23-55 (in Polish with English summary).

KUČERA J., VÁŇA J. \& HRADÍLEK Z. 2012. Bryophyte flora of the Czech Republic: updated checklist and Red List and a brief analysis. Preslia 84: 813-850.

LiMPRICHT K. G. 1873. Nachträge zu J. Milde: Bryologia Silesiaca, 1869. Jahresber. Schles. Ges. Vaterl. Cult. 50: 124-140.

LiMPRICHT K. G. 1904. Die Laubmoose Deutschlands, Oesterreichs und der Schweitz unter Berücksichtigung der übrigen Länder Europas u. Sibiriens. In: Dr L. Rabrnhorst's Kryptogamen-Flora von Deutschlands, Oesterreichs und der Schweitz. 2 Aufl. 4(3) - Hypnaceae u. Nachträge, Synonymen-Register $u$. Literatur-Verzeichniss. Eduard Kummer, Leipzig. 
LisowSKI S. 1956. Mosses of the Western Bieszczady. Prace Komis. Biol. 17(3): 1-85 (in Polish with English summary).

LisOWSKI S. \& KORNAŚ J. 1966. Mosses of the Gorce Mts. (Polish Western Carpathians). Fragm. Flor. Geobot. 12(1): 41-111 (in Polish with English summary).

MEINUNGER L. \& SCHRÖDER W. 2007. Verbreitungsatlas der Moose Deutschlands. Band 3. Regensburgische Botanische Gesellschaft, Regensburg.

MiLdE J. 1869. Bryologia Silesiaca, Laubmoos-Flora von Nord- und Mittel-Deutschland, unter besonderer Berücksichtigung Schlesiens und mit Hinzunahme der Floren von Jütland, Holland, der Rheinpfalz, von Baden, Franken, Böhmen, Mähren und der Umgegend von München. Arthur Felix, Leipzig.

OCHYRA R. 1988. Spostrzeżenia briologiczne z Mierzei Helskiej (Bryological observations from the Hel Peninsula (Baltic coast, the Gulf of Gdańsk). Badan. Fizjogr. Polsk. Zachodn., B 37: 167-175 (in Polish with English summary).

Ochyra R. \& Stebel A. 2008. Mosses of the Małe Pieniny Range (Polish Western Carpathians). In: A. STEBEL $\&$ R. OCHYRA (eds), Bryophytes of the Polish Carpathians. pp. 75-141. Sorus, Poznań.

OCHYRA R. \& SZMAJDA P. 1981. La cartographie bryologique en Pologne. - In: J. SZWEYKOWSKI (ed.), New perspectives in bryotaxonomy and bryogeography. Uniwersytet im. Adama Mickiewicza w Poznaniu Seria Biologia 20: 105-110.

REHMANN A. 1865. Versuch einer Aufzählung der Laubmoose von Westgalizien. Verh. Zool.-Bot. Ges. Wien 15: 461-484.
SteBEL A. 1997. Bryophytes of the Rybnik Coal Dictrict (S Poland). Fragm. Flor. Geobot. Ser. Polonica 4: 121-233 (in Polish with English summary).

Stebel A. 2008. Mosses of the Kotlina Żywiecka Basin (Western Carpathians). In: A. STEBEL \& R. OCHYRA (eds), Bryophytes of the Polish Carpathians. pp. 11-74. Sorus, Poznań.

Stebel A. 2010. Mosses of the Beskid Mały Range (Western Carpathians). Centrum Dziedzictwa Przyrody Górnego Ślaska w Katowicach. Materiaty Opracowania 11: 1-142.

SteBel A. \& OChYRA R. 2000. The moss flora of the Magura National Park in the Beskid Niski Range (Western Carpathians). Fragm. Florist. Geobot. Ser. Polonica 7: 229263 (in Polish with English summary).

Stebel A., Ochyra R. \& VonČina G. 2010. Mosses of the Pieniny Range (Polish Western Carpathians). Sorus, Poznań.

SzAFrAN B. 1961. Flora polska. Rośliny zarodnikowe Polski i ziem ościennych. 2. Państwowe Wydawnictwo Naukowe, Warszawa.

SzAFRAN B. 1968. List of the mosses collected in the environs of Przemyśl (South-eastern Poland). Fragm. Florist. Geobot. 14(2): 237-239 (in Polish with English summary).

SZUKALSKA D. 2007. Plant communities on dead trees in forest of northern slopes of Babia Góra (Beskidy Mts.). Bogucki Wydawnictwo Naukowe, Poznań. 